\title{
artigo
}

Oliveira, S.P.; Lucena Silva, L.H.; Brito, J.D.; Ferreira, F.B.M.; Carvalho, C.B; Parga, L.D.; Ferreira, R.L.; Gaspar, M.A.R.; Costa, C.W.M.; Barros, L.A.A.; Neves, A.C.F.B.; Lima, S.F.;

Matriz de indicadores de hanseníase em pinheiro - Maranhão de 2015 a 2017

\section{Matriz de indicadores de hanseníase em pinheiro - Maranhão de 2015 a 2017}

\author{
Matrix of leprosy indicators in pinheiro - Maranhão 2015 to 2017
}

Matriz de indicadores de lepra en pinheiro - Maranhão 2015 a 2017

\begin{abstract}
RESUMO
Objetivos: discutir o panorama da doença em Pinheiro - MA e ponderar novas perspectivas acerca do cuidado em saúde referente à patologia, sob a ótica da interprofissionalidade. Métodos: Realizou-se um estudo transversal, descritivo e retrospectivo, entre 2015 e 2017, utilizando-se arquivos do departamento de controle e tratamento da Hanseníase localizado no Centro de Especialidades Médicas de Pinheiro (CEMP). 0 programa Stata, versão 14 foi utilizado para análise das variáveis. Resultados: 0 perfil epidemiológico do município demostrou prevalência de homens 58 (52,7\%), entre 40 - 59 anos 28(25,45\%), com ensino fundamental incompleto e classificação terapêutica multibacilar prevalente $60(54,5 \%)$. Maioria dos pacientes teve alta no período de análise $66(60 \%)$. A avaliação dermatoneurológica está subnotificada. Conclusão: A situação mapeada aponta falhas nas estratégias de enfrentamento da hanseníase que poderiam ser reformulado através de trabalho interprofissional, colaborativo e estruturado.
\end{abstract}

DESCRITORES: Hanseníase; Indicadores; Epidemiologia; Educação Interprofissional.

\section{ABSTRACT}

Objectives: to discuss the panorama of the disease in Pinheiro - MA and to consider new perspectives on health care related to pathology, from the perspective of interprofessionality. Methods: A cross-sectional, descriptive and retrospective study was carried out between 2015 and 2017, using files from the Hansen's disease control and treatment department located at the Centro de Especialidades Médicas de Pinheiro (CEMP). The Stata program, version 14 was used to analyze the variables. Results: The epidemiological profile of the municipality showed a prevalence of men $58(52.7 \%)$, between 40 - 59 years old $28(25.45 \%)$, with incomplete elementary education and prevalent multibacillary therapeutic classification 60 (54.5\%). Most patients were discharged during the analysis period $66(60 \%)$. The dermatoneurological evaluation is underreported. Conclusion: The mapped situation points out flaws in the strategies for coping with leprosy that could be reformulated through interprofessional, collaborative and structured work.

DESCRIPTORS: Leprosy; Indicators; Epidemiology; Interprofessional Education.

\section{RESUMEN}

Objetivos: discutir el panorama de la enfermedad en Pinheiro - MA y considerar nuevas perspectivas sobre la atención de la salud relacionada con la patología, desde la perspectiva de la interprofesionalidad. Métodos: se realizó un estudio transversal, descriptivo y retrospectivo entre 2015 y 2017, utilizando archivos del departamento de control y tratamiento de la enfermedad de Hansen ubicado en el Centro de Especialidades Médicas de Pinheiro (CEMP). Se utilizó el programa Stata, versión 14 para analizar las variables. Resultados: El perfil epidemiológico del municipio mostró una prevalencia de hombres $58(52,7 \%)$, entre 40 - 59 años 28 (25,45\%), con educación básica incompleta y clasificación terapéutica multibacilar prevalente 60 (54,5\%). La mayoría de los pacientes fueron dados de alta durante el período de análisis 66 (60\%). La evaluación dermatoneurológica no se informa. Conclusión: La situación cartografiada señala fallas en las estrategias de afrontamiento de la lepra que podrían reformularse mediante un trabajo interprofesional, colaborativo y estructurado.

DESCRIPTORES: Lepra; Indicadores; Epidemiología; Educación Interprofesional.

RECEBIDO EM: 30/01/2021 APROVADO EM: 16/02/2021

\section{Sanny Pinheiro Oliveira}

Acadêmica de Medicina. Universidade Federal do Maranhão - Campus Pinheiro.

ORCID: 0000-0002-4197-4368 
Laís da Hora Lucena Silva

Acadêmica de Medicina. Universidade Federal do Maranhão - Campus Pinheiro.

ORCID: 0000-0003-4558-6052

\section{Jundson Dias Brito}

Acadêmico de Enfermagem. Universidade Federal do Maranhão - Campus Pinheiro.

ORCID: 0000-0003-4741-5265

\section{Fernanda Bernadeth Monteiro Ferreira}

Acadêmica de Educação Física. Universidade Federal do Maranhão - Campus Pinheiro.

ORCID: 0000-0002-7569-9042

\section{Clarice Borges Carvalho}

Acadêmico de Enfermagem. Universidade Federal do Maranhão - Campus Pinheiro.

ORCID: 0000-0001-6457-7923

\section{Larissa Dias Parga}

Acadêmico de Enfermagem. Universidade Federal do Maranhão - Campus Pinheiro.

ORCID: 0000-0002-6590-2502

\section{Rafaella Lopes Ferreira}

Acadêmica de Medicina. Universidade Federal do Maranhão - Campus Pinheiro.

ORCID: 0000-0001-9015-0560

\section{Maria Augusta Ribeiro Gaspar}

Acadêmica de Medicina. Universidade Federal do Maranhão - Campus Pinheiro.

ORCID: 0000-0001-5056-3301

\section{Cíntia Wélia Moraes Costa}

Terapeuta Ocupacional. Secretaria Municipal de Saúde e Saneamento Básico de Pinheiro-(SEMUS).

ORCID: 0000-0001-7119-4939

\section{Lidiane Andréia Assunção Barros}

Docente do Curso de Enfermagem. Universidade Federal do Maranhão, Campus Pinheiro. Enfermeira. Mestra em Enfermagem - UFMA. ORCID: 0000-0002-1614-3845

\section{Ariane Cristina Ferreira Bernardes Neves}

Docente do Curso de Medicina. Universidade Federal do Maranhão, Campus Pinheiro. Enfermeira. Doutora em Saúde Coletiva - UFMA. ORCID: 0000-0002-5258-1172

\section{Sara Fiterman Lima}

Docente do Curso de Medicina. Universidade Federal do Maranhão, Campus Pinheiro. Enfermeira. Doutora em Ciências da Saúde - UFMA.

ORCID: 0000-0002-8393-5025

\section{INTRODUÇÃO}

A hanseníase é estabelecida pela Organização Mundial da Saúde (OMS) como um problema de saúde pública, por causar incapacidade física, social e econômica. Uma das características da doença é o tropismo do Mycobacterium leprae por nervos periféricos, resultando em dano neural. A doença pode evoluir de forma lenta e silenciosa, com diminuição da sensibilidade térmica, dolorosa, e tátil ${ }^{1}$.

De acordo com Ribeiro ${ }^{2}$, a prevalência de casos de hanseníase no Brasil no ano de 2015 foi de 1,01/10 000 habitantes. Os coeficientes de prevalência mais elevados foram registrados entre os anos de 2005 e 2009. Desde 2005, o coeficiente de prevalência do país permaneceu classificado como médio (de 1,00 a 4,99/10 000 habitantes). Apesar da tendência nacional de diminuição da prevalência, esse comportamento não foi observado nas regiões Norte, Nordeste e Centro-Oeste apresentando coeficiente de prevalência acima da média nacional no ano de 2015 . Os estados responsáveis pelos altos índices de prevalência nessas regiões no ano de 2015 foram Mato Grosso, no Centro-Oeste (7,75/10 000 habitantes); Tocantins, no Norte (4,2/10 000 habitantes); e Maranhão, no Nordeste (3,76/10 000 habitan- 


\section{artigo}

Oliveira, S.P.; Lucena Silva, L.H.; Brito, J.D.; Ferreira, F.B.M.; Carvalho, C.B; Parga, L.D.; Ferreira, R.L.; Gaspar, M.A.R.; Costa, C.W.M.; Barros, L.A.A.; Neves, A.C.F.B.; Lima, S.F. Matriz de indicadores de hanseníase em pinheiro - Maranhão de 2015 a 2017

tes). As regiões Sudeste e Sul não tiveram registro de casos de hanseníase entre 2005 e 2008. Nos demais anos, o coeficiente de prevalência ficou abaixo da média nacional nessas regiões. ${ }^{2}$

O Brasil ocupa a segunda posição mundial em número de casos novos notificados, perdendo apenas da Índia. No Maranhão, em 2011, foram detectados 56 casos por 100.000 habitantes, caracterizando-o como estado hiperendêmico. Suas taxas médias de detecção ultrapassam a média do Nordeste e do Brasi $1^{3,4}$.

Partindo do princípio de que não há uma forma eficiente de se proteger da doença e compreendendo que sua transmissão está associada a um menor nível socioeconômico, justifica-se a prevalência da doença em países em desenvolvimento5. A doença está implicada numa baixa mortalidade, contudo alta morbidade, principalmente em função das sequelas neurológicas e dos estigmas sociais $^{6,7,8,9}$.

O Projeto PET SAÚDE - Interprofissionalidade de Pinheiro no Maranhão tem como um de seus grupos tutoriais voltado para a temática da Hanseníase, que possui altas taxas de incidência e prevalência na região. Entende-se por educação interprofissional (EIP), quando estudantes de duas ou mais profissões aprendem sobre os outros e entre si para possibilitar a colaboração eficaz e melhorar os resultados na saúde ${ }^{10}$. Neste sentido, compõem objetivos desse estudo discutir o panorama da doença em Pinheiro - MA e ponderar novas perspectivas acerca do cuidado em saúde referente à patologia, sob a ótica da interprofissionalidade.

\section{MÉTODOS}

Realizou-se um estudo transversal, des-

\section{hanseníase em Pinheiro - Maranhão, no período de 2015 a 2017.}

$$
\text { VARÁVEIS }
$$

Idade

$0-15$ anos
$16-39$ anos
$40-59$ anos
60 anos ou mais

0

\section{Sexo}

Masculino

Feminino

\section{Escolaridade}

Não informado

Analfabeto

Fundamental inc.

Fundamental comp.

Médio incompleto

Médio completo

Graduação

\section{Classificação}

Paucibacilar

Multibacilar

Total de casos

\section{3}

14

18

14

32

19

15

8

16

1

1

10

0

17

31

51
0

9

7

7

15

8

5

0

12

0

3

3

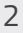

5

18

23

Fonte: Fichas da vigilância epidemiológica de Pinheiro/MA.

critivo e retrospectivo, utilizando-se dados de 2015 a 2017, disponíveis nos arquivos do departamento de controle e tratamento da Hanseníase localizado no Centro de Especialidades Médicas de Pinheiro (CEMP). As variáveis do estudo foram agrupadas em nove categorias: idade, sexo, escolaridade, classificação, abandono, alta, óbito, transferências e avaliação neurológica.

Para a coleta dos dados, utilizou-se um formulário específico com informações compreendidas nas fichas de vigilância dos pacientes, desenvolvido pelos integrantes do Grupo Pet-Hanseníase.

A população do estudo contou com 91 pacientes notificados entre os anos de 2015 (17 casos), 2016 (51 casos) e 2017 (23 casos). A definição das formas clínicas foi feita a partir das características relacionadas pelo Ministério da Saúde (MS) do Brasil, em paucibacilares e multibacilares.

Para cálculo da referência, utilizou-se a população de Pinheiro - Maranhão, segundo o IBGE 2018, totalizando 82.990 pessoas. Todas as taxas foram equivalentes para 100.000 mil habitantes. Para cálculo da incidência, excluíram-se os casos de óbito e de transferência intermunicipal. A análise foi realizada através de estatística descritiva simples e comparativa, utilizando o programa Stata, versão 14.

O presente estudo obedeceu aos trâmites éticos estabelecidos pela Resolução do Conselho Nacional de Saúde 466/2012, sendo aprovado pelo Comitê de Ética em Pesquisa da Universidade Federal do Maranhão sob o CAAE $\mathrm{n}^{\circ}$ 29652520.0.0000.5087 e Parecer $\mathrm{n}^{\circ}$ 3.938.591.

\section{RESULTADOS}

A hanseníase apresentou prevalência de 110 casos para 100 mil habitantes, no município, nos anos de 2015 a 2017. Quanto à incidência, observou-se 20 casos, 57 casos e 23 casos nos respectivos anos de análise. A situação epidemiológica da hanseníase encontra-se disposta na tabela 1 .

Quanto à idade, tem-se a prevalência da faixa etária dos maiores de 15 anos 
em todos os anos analisados. Em 2016 tem-se o pico da faixa de 40 - 59 anos, e duas idades não foram notificadas. $\mathrm{O}$ sexo masculino foi mais prevalente em todos os anos analisados, 11 (64,79\%), $32(62,74 \%)$ e $15(65,21 \%)$ casos em ordem crescente dos anos pontuados. No que se refere à escolaridade, a maior prevalência é de "ensino fundamental incompleto”, e não há registros em 2015. Pontua-se que o número de casos 20 nos anos analisados. O número de graduados com hanseníase foi de apenas a formação acadêmica cumpre função inversa quando relacionada proporcionalmente ao número total de casos. (27\%) não informados é significativo dois casos em 2017. Em números gerais

A frequência dos multibacilares é maior em todos os anos pesquisados $11(64,79 \%), 31(64,38 \%)$ e $18(78,26 \%)$ em 2015, 2016 e 2017, respectivamente. Dois casos quanto à classificação terapêutica não foram registrados em 2016. A taxa de alta prevalece quanto à evolução terapêutica de hanseníase no período 13 (76,47\%), $39(76,47 \%)$ e $14(60,86 \%)$ em ordem crescente dos anos. Duas evoluções não foram informadas nos anos pesquisados. Relata-se um óbito em 2016 e um em 2017. Por fim consideram-se as taxas de abandono significativas ao longo do espaço temporal $12(13,18 \%)$, frente à transmissão da doença e sua evolução.

Com relação à presença de incapacidades físicas, observa-se que o número

Gráfico 1. Seguimento terapêutico de hanseníase em Pinheiro - Maranhão, no período de 2015 a 2017

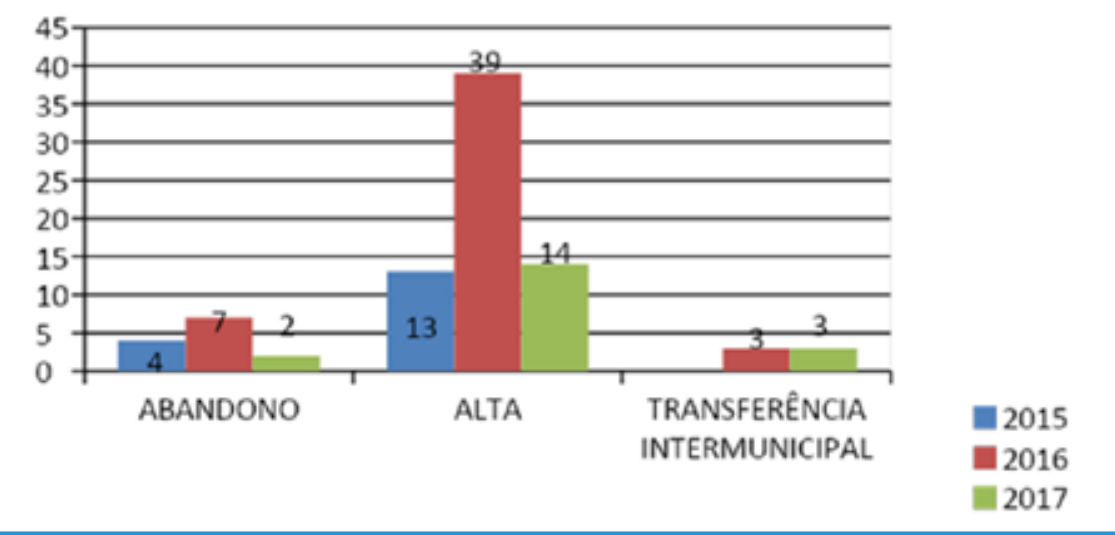

Fonte: Fichas da vigilância epidemiológica de Pinheiro/MA

Gráfico 2. Grau de incapacidade por hanseníase em Pinheiro - Maranhão, de 2015 a 2017

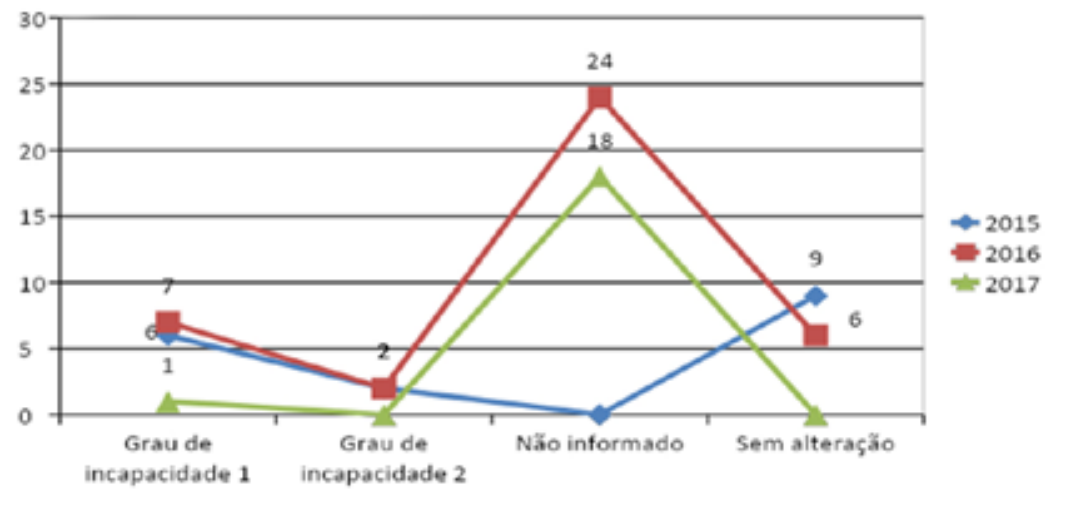

Fonte: Fichas da vigilância epidemiológica de Pinheiro/MA de casos não informados é prevalente 24 (61,53\%), 18 (78,26\%) nos anos de 2016 e 2017 (Gráfico 2). Essa categoria não foi relatada em 2015. Dentre as categorias que investigam o grau de incapacidade, destaca-se o "Grau de incapacidade 1" com $6(35,29 \%), 7$ (7,39\%) e 1 (5,26\%). Dentre as alterações relatadas pontuam-se: sensibilidade térmica e dolorosa ausente ou diminuída em qualquer parte do corpo encontrada em cinco casos, acuidade visual em 3 casos, espessamento fibular em 3 casos, dor e parestesia dos membros em 3 casos e um caso de alopecia nas manchas.

\section{DISCUSSÃO}

O município de Pinheiro apresentou 110 casos de hanseníase para 100.000 no período estudado, dados que ultrapassam a prevalência brasileira de 14,8 casos para 100.000 habitantes, em 201810. Todos os territórios em análise ferem a meta de eliminação da hanseníase proposta pela OMS (menos de 10 casos por cem mil habitantes) $)^{11}$.

Mostrou-se prevalência da população masculina no estudo. Este fato pode ser explicado pela negligência com a saúde, somado a maior exposição em ambientes de risco, característicos dessa população ${ }^{12}$.

Em consonância com a produção literária os casos se concentram na faixa etária dos maiores de 15 anos, sem significância entre as classes $^{13}$. Por atingir a população economicamente ativa, frente à possibilidade de incapacidades físicas e alterações neurológicas, estipulam-se prejuízos à economia. Em 2015 há três casos relatados entre os menores de 15 anos. A ocorrência de hanseníase em crianças pode ser considerada um indicador da prevalência da doença na população geral e sua detecção é importante para determinar a gravidade da endemi $a^{14}$.

Indivíduos de baixa escolaridade prevaleceram no estudo, apontando necessidade de focalizar ações educativas para essa classe. Essa concentração está atrelada às condições de vida, grau de exposição e acesso a rede de saúde. A porcentagem significativa de não preenchimento dessa ca- 


\section{artigo}

Oliveira, S.P.; Lucena Silva, L.H.; Brito, J.D.; Ferreira, F.B.M.; Carvalho, C.B; Parga, L.D.; Ferreira, R.L.; Gaspar, M.A.R.; Costa, C.W.M.; Barros, L.A.A.; Neves, A.C.F.B.; Lima, S.F.

Matriz de indicadores de hanseníase em pinheiro - Maranhão de 2015 a 2017

tegoria pode alterar a disposição e análise dos dados em relação a essa variável.

O predomínio de multibacilares está em concordância a artigos similares. Esses pacientes são, em geral, os mais suscetíveis à doença e a maior taxa de transmissão da hanseníase, o que reitera necessidade de identificação e tratamento dessa população ${ }^{15}$.

Apesar do prevalecimento de alta clínica, a significativa taxa de abandono pressupõe retomada do ciclo de propagação em uma sociedade fragilizada quanto à saúde e à economia. A subnotificação quanto ao grau de incapacidade física pontua falta de investigação para essas alterações ou não relato aos órgãos competentes seja por falta de equipamento e/ou prática investi- gativa, seja por morosidade de notificação, frente ao acúmulo de tarefas.

Considerando os baixos níveis socioeconômicos do município somados a negligência característica da patologia que se interseccionam e potencializam a endemicidade disposta em Pinheiro - MA. Nesse sentido, o trabalho em equipe através da Interprofissionalidade surge como estratégia para a reformulação do método de trabalho pretendendo melhor qualidade nos serviços de saúde da Atenção Básica, formação profissional e procurando focar as relações entre saberes e práticas acadêmicas, profissionais e comunitárias por meio do trabalho colaborativo, efetivo e resolutivo ${ }^{16,17}$.

\section{CONCLUSÃO}

Conclui-se que o município de Pinheiro no Maranhão é hiperendêmico em relação à hanseníase. Em geral os pacientes são homens, maiores de 15 anos, de baixa escolaridade, multibaciliares com seguimento terapêutico prevalente para alta. Diante deste cenário, evidencia-se a necessidade de facilitar o acesso ao diagnóstico e ao tratamento precoce, por meio da reestruturação do programa de controle à hanseníase com enfoque na união de forças para uma abordagem ampla, que valorize os diferentes papeis envolvidos, sejam eles o da família, comunidade, usuário, equipe de saúde e acadêmica. -

\section{REFERÊNCIAS}

1. Santos AR; Ignotti E. Prevenção de incapacidade física por hanseníase no Brasil: análise histórica. Ciência \& Saúde Coletiva. 2020;25:3731-3744.

2. Ribeiro MDA, Silva, JC, Oliveira SB. Estudo epidemiológico da hanseníase no Brasil: reflexão sobre as metas de eliminação. Rev Panamericana de Salud Pública. 2018;42(42):01-07.

3. Santos GR et al. Prevalência de hanseníase em São Luís Maranhão entre os anos de 2013 a 2015. J. nurs. Health.2018;8(2):01-13.

4. Brasil. Ministério da Saúde, Diretrizes para vigilância, atenção e eliminação da hanseníase como problema de saúde pública. Manual técnico-operacional Brasília: MS; 2016.

5. Goiabeira YNLA, Rolim ILTP, Aquino DMC, Santos LH, Lima ABS, Soeiro VMS. Programa de controle da hanseníase em capital hiperendêmica: uma avaliação operacional. Rev baiana enfermagem. 2018;32(25144):01-09.

6. Gomes FG, Frade MAC, Foss NT. Úlceras cutâneas na hanseníase: perfil clínico-epidemiológico dos pacientes. An Bras Dermatol. 2007;82(5):433-7.

7. Costa JC. Perfil epidemiológico da hanseníase no Brasil nos anos de 2014 a 2016. Tubarão: Universidade do Sul de Santa Catarina, 2018. Trabalho de Conclusão de Curso em Medicina.

8. Brasil, Ministério da Saúde, Secretaria de Vigilância em Saúde. Boletim Epidemiológico. Situação epidemiológica da hanseníase no Brasil - análise de indicadores selecionados na última década e desafios para eliminação. Bol Epidemiol. 2013;44(11):1-12.

9. Brasil, Ministério da Saúde, Secretaria de Vigilância em Saúde, Departamento de Vigilância em Doenças Transmissíveis. Plano integrado de ações estratégicas de eliminação da hanseníase, filariose, esquistossomose e oncocercose como problema de saúde pública, tracoma como causa de cegueira e controle de geohelmintíases: plano de ação 2011-2015. Brasilia: MS/SVS/DVDT; 2012.

10. Costa JC. Perfil epidemiológico da hanseníase no Brasil nos anos de 2014 a 2016. Tubarão: Universidade do Sul de Santa Catarina, 2018. Trabalho de Conclusão de Curso em Medicina.

11. Anchieta JJS et al. Análise da tendência dos indicadores da hanseníase em estado brasileiro hiperendêmico, 2001-2015. Rev Saude Publica. 2019;53-61.

12. Sarmento APV et al. Perfil epidemiológico da hanseníase no período de 2009 a 2013. Unversidade Estadual de Montes Claros. Montes Claros, MG. 2015.

13. Lima HMN et al. Perfil epidemiológico dos pacientes com hanseníase atendidos em Centro de Saúde em São Luís, M. Departamento de Enfermagem do Centro Universitário do Maranhão (UNICEUMA), São Luis, MA. 2010.

14. Duarte MTC, Ayres JA, Souza MM, Simonetti JP. Perfil socioeconômico e demográfico de portadores de hanseníase atendidos em consulta de enfermagem. Rev Latino-am Enfermagem setembro-outubro; 15(número especial) www.eerp.usp.br/rlae. 2007

15. Silva, $L$ de $A$ et al. Aspectos epidemiológicos dos casos de hanseníase em um município no interior do maranhão. Centro Universitário da Uninovafapi. Teresina, PI. 2017.

16. Silva JAM, Peduzzi M, Orchard C, Leonello VM. Educação interprofissional e prática colaborativa na Atenção Primária à Saúde*. Rev da Escola de Enfermagem da Usp. 2015;49(2):16-24.

17. Lima WLS et al. Percepções sobre a importância da educação interprofissional na formação dos profissionais de saúde: relato de experiência de um grupo do Pet-Saúde/Interprofissionalidade. Rev Saúde Coletiva Uefs. 2020;10(2):82-89. 